\title{
An Architectural Model to Promote User Awareness on the Web
}

\author{
Claudio O. Gutiérrez ${ }^{1}$, Luis A. Guerrero², and César A. Collazos ${ }^{3}$ \\ ${ }^{1}$ Departament of Computer, Universidad de Magallanes, Avenida Bulnes 01855 \\ Punta Arenas, Chile \\ cjoelg@ona.fi.umag.cl \\ ${ }^{2}$ Departament of Computer Science, Universidad de Chile, Blanco Encalada 2120 \\ Santiago, Chile \\ luguerre@dcc.uchile.cl \\ ${ }^{3}$ Departament of Compute Science, Universidad del Cauca, Campus Tulcan \\ Popayan, Colombia \\ ccollazo@unicauca.edu.co
}

\begin{abstract}
Every day more people use the Web as a tool to collaborate and to do work group. Nevertheless, as collaborative tool, the Web does not have one of the most important features of this kind of tools: a user awareness system. User awareness gives the possibility to receive feedback about people that could be interested in the same concern and give the possibility to share knowledge about the sources or Web pages that they consider relevant.
\end{abstract}

\section{Introduction}

The main focus of this paper is to propose a new architectural model on the Web to promote user awareness. The primary goal of this model is to offer the necessary mechanisms to the final users of the Web in order to give them the possibility of establish communication and collaboration with their couples. Similar situations happen in some virtual environments (see for example [1]). These awareness mechanisms are based on the delivery of historical information of those who have visited the same Web sites. The information provided by the system contains data surrendered by people that visited these places. These data assure the possibility to establish further communication with people that already visited those places. This information is compound basically for data such as name, e-mail, comments and interests, to mention the most relevant. Our architecture is implemented in Java and one of the main advantages is that we do not alter the current operation of the Web.

Most of the technology used to present information tipically focus on the use of direct audio and video connection as an aid to collaboration among remotely located people. The technologies to promote the use of the audio and the video generally require of high-speed dedicated connections. However most of the people that have used the Web have different connection types, with different access speeds and where in general the connections are not stable. The collaboration that takes place is basically asynchronous and the people have a few opportunities to meet with another. Moreover the browsers are mainly tools focused to lend services to a single user, 
which even increases the invisibility of them. On the other hand, we could notice the Web as a big share workspace where a lot of people is dedicated to reading activities where shared objects are in the same site. In these context the activities of other people could be an important factor respect to the interest that causes a site or a specific Web page. In other words, we are giving importance to one of the entities most important of the Web: the final users. The model we propose provides a kind of awareness where the final users receives feedback about people that are interested in the same topic.

\section{Architecture of the Proposed Model for User Awareness}

Our main objective is to provide Web users with awareness about other users. The interaction among our model components is over TCP/IP communication protocol.

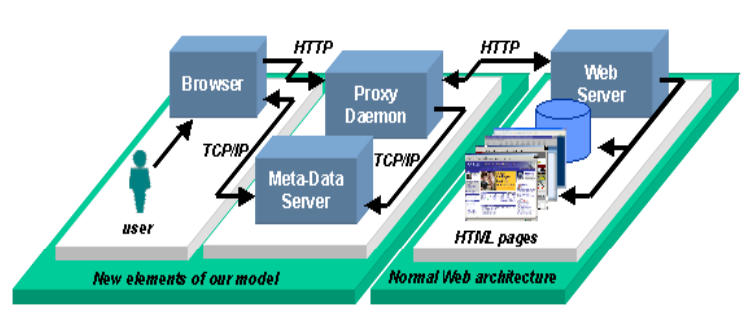

Fig. 1. (a) The Model Architecture;

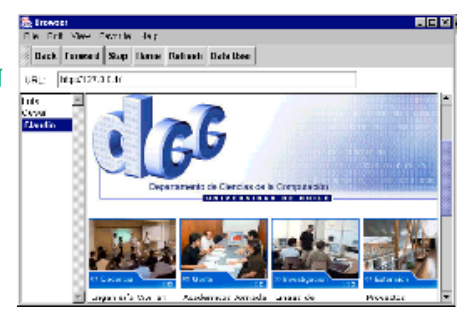

(b) The Browser Interface

Figure 1a shows our model design, which maintains the components and the current operation of the Web architecture. However our proposal points to extend this architecture, adding components that provide user's awarenes in the Web. The advantage of this schema is that the model does not modify the components neither the operation of the conventional architecture of the Web. The figure $1 \mathrm{~b}$ depicts our sample browser. This one is composed of three main panels: the left hand side shows the names of people that are already registered in the system and had visited this page recently. The right side shows the current Web page the user is browsing, and on the top level there is a normal toolbar that support standard functions of the conventional Web browsers.

\section{Reference}

[1] Shapiro, E. Virtual Places - A Foundation for Human Interaction. Proceedings of WWW2, the 2nd International World Wide Web Conference, Chicago, IL. May 1994. 